\title{
Análisis Envolvente de Datos y Cálculo Multivariado para Valorar, Clasificar y Predecir la Eficiencia Productiva y de Innovación de las Empresas del Sector Químico.
}

\author{
Efraín De La Hoz ${ }^{(1) \star}$, Tomás Fontalvo ${ }^{(1)}$ y Ludis López ${ }^{(2)}$ \\ (1) Universidad de Cartagena, Facultad de Ciencias Económicas, Programa Administración Industrial. Campus Piedra \\ de Bolívar, Cartagena. Colombia, (e-mail: tfontalvoh@unicartagena.edu.co, edelahozg@unicartagena.edu.co). \\ (2) Universidad Simón Bolívar, Facultad de Ingeniería, Programa de Ingeniería Industrial, Colombia. \\ (e-mail: lulopez@unisimonbolivar.edu.co). \\ ${ }^{*}$ Autor a quien debe ser dirigida la correspondencia
}

Recibido Dic. 3, 2018; Aceptado Ene. 30, 2019; Versión final Mar. 25, 2019, Publicado Oct. 2019

\begin{abstract}
Resumen
Se desarrolló un método que permitió establecer criterios de valoración, clasificación y predicción para evaluar la eficiencia productiva y de innovación de las empresas del sector químico de Barranquilla. Se recolectó información asociada a variables de clima laboral, gestión de la información, gestión del conocimiento, gestión de la productividad y la innovación. Seguidamente se validó los resultados con el análisis discriminante y se modelaron procesos de pronóstico y predicción de la eficiencia de las empresas del sector con las redes neuronales artificiales. Los resultados muestran que la eficiencia promedio en el sector es del $52,9 \%$, con 6 empresas clasificadas como eficientes. Con las técnicas de análisis discriminante multivariado, se pudo determinar la calidad de clasificación lográndose un 92,6 \% de clasificación correcta. Así mismo el modelo de redes neuronales seleccionado generó una precisión de clasificación de 98,82\%, 95,78\% y 94,28\% para las muestras de entrenamiento, prueba y reserva, lo que evidencia la relevancia del modelo de clasificación. Se concluye que las variables analizadas son significativas para discriminar la eficiencia productiva y de innovación.
\end{abstract}

\section{Data Envelopment Analysis and Multivariate Calculus to Assess, Classify and Predict the Productive Efficiency and Innovation of Companies in the Chemical sector}

\begin{abstract}
A method to establish assessment, classification and prediction criteria to evaluate the productive efficiency and innovation of the companies in the chemical sector of Barranquilla was developed. Concepts of data envelopment analysis, discriminant analysis and artificial neural networks were used. Information associated with variables of labor climate, information management, knowledge management, productivity management and innovation, were collected. The results were then validated with the discriminant analysis and forecasting processes and prediction of the efficiency of the companies in the sector with artificial neural networks were modeled. The results show that the average efficiency in the sector is $52.9 \%$, with 6 companies classified as efficient ones. With the multivariate discriminant analysis techniques, the classification quality could be determined, achieving a 92.6\% correct classification. Likewise, the selected neural network model generated a classification accuracy of $98.82 \%, 95.78 \%$ and $94.28 \%$ for the training, test and reserve samples, which shows the relevance of the classification model. It is concluded that the analyzed variables are significant to discriminate productive efficiency and innovation.
\end{abstract}




\section{INTRODUCCIÓN}

El sector químico es uno de los sectores más dinámicos de la economía a nivel nacional e internacional, de igual forma en la Costa Atlántica y específicamente en la ciudad de Barranquilla, este juega y ha jugado un papel determinante para la consolidación y desarrollo de la economía del caribe colombiano. Así mismo las condiciones cambiantes de la economía, como consecuencia de la globalización a nivel internacional ha generado la necesidad de analizar diferentes variables empresariales como la gestión del clima laboral (GCL), la gestión del conocimiento $(\mathrm{GC})$ y la gestión de la información (Gl) y su incidencia en la gestión de la productividad (GP) y la gestión de la innovación (GIN) en las empresas del sector químico de la ciudad de Barranquilla. De lo anterior, en la presente investigación, se plantean las siguientes preguntas problemas ¿Cómo clasificar las variables asociadas al estudio al modelo Análisis Envolvente de Datos?, ¿Cuál es el nivel de eficiencia y el desempeño de las diferentes empresas del sector Químico de Barranquilla en términos de la gestión de productividad e innovación?, ¿Existe correlación de causalidad entre las variables de entrada o recursos con las variables de salida asociadas a los resultados para generar eficiencia?, ¿Qué modelo de cálculo multivariado utilizar para clasificar empresas eficientes y no eficientes de acuerdo al modelo DEA utilizado?, ¿Cómo y con qué método de cálculo multivariado se puede clasificar y pronosticar la pertenencia del grupo objeto de estudio a empresas eficientes y no eficientes?, ¿De qué manera predecir una nueva empresa del sector químico en eficiente o no eficiente? Lo anterior generó la necesidad de establecer una metodología de valoración, clasificación y predicción de la eficiencia productiva y de innovación de las empresas del sector Químico a través de la utilización de herramientas de análisis envolvente de datos y las técnicas de cálculo multivariado, redes neuronales y análisis discriminante.

Según López (2013), la productividad, comprende la generación de riqueza sustentada por acciones éticas y morales que deben tener un impacto social en armonía con el medio ambiente. Así mismo establece que la productividad y la eficiencia son dos aspectos que coexisten y en la práctica son inseparables, por lo que el concepto de productividad implica la eficiencia. De esta manera se puede hablar de "eficiencia productiva" o "productividad eficiente". Por otra parte, la innovación empresarial, requiere el compromiso y la voluntad organizacional para la conformación de equipos de trabajo comprometidos en la búsqueda de soluciones a los problemas de la empresa para lo cual asume riesgos y se busca con productos y servicios creativos la satisfacción de las necesidades, lo que le permite sorprender al mercado y la competencia logrando el liderazgo (Martínez, 2010). En este sentido, Segredo (2013) resalta la importancia que tiene el clima organizacional o clima laboral en las organizaciones como factor para el mejoramiento continuo del ambiente laboral, por lo que se constituye en pieza clave para el desarrollo de la organización. Su análisis profundo, diagnóstico son necesarios para una adecuada gestión del cambio, por lo que su mejora impacta directamente de manera particular el comportamiento humano y de manera general, el espíritu de la organización. De la misma manera, Gan y Trigine (2012), comparten la importancia del clima laboral como factor determinante en la dinámica empresarial, el cual es afectado por múltiples factores como la normatividad interna de la organización, las condiciones laborales, la actitud de las personas que conforman el equipo de trabajo entre otros factores. Chiang, et al (2010) establece lo relaciona con atributos o conjunto de atributos del ambiente laboral, su importancia en la organización, radica en las personas, debido a su impacto en la interacción laboral, conducta como en sus sentimientos. Por su parte, Serrate (2014) resaltan la importancia del análisis del clima laboral para los directivos de una organización dado que revelan los aspectos que afectan favorable o desfavorablemente la dinámica de trabajo.

De la Peña (2015) conceptúa que la información es un activo intangible de la organización, por lo cual constituye un bien económico que puede ser comparable con una patente o una licencia. De Pablos, et al. (2006) reconocen que la información y el conocimiento, son factores mediante las cuales la organización conoce las necesidades de la sociedad, así como la dinámica del mercado encabezada por los competidores. Los sistemas de información, no solo están diseñados para gestionar la información y el conocimiento en las organizaciones, sino también para contribuir en el mejoramiento de los procesos de negocio y la toma de decisiones impactando su rentabilidad. Según Alegra (2004), la gestión del conocimiento consiste en un conjunto de técnicas, herramientas y prácticas cuyo propósito es la creación, distribución, almacenamiento y aplicación del conocimiento, la cual proporciona competencias distintivas que proporcionan ventajas competitivas para la organización. Cegarra y Martínez (2017), conceptúan que el conocimiento ha pasado a ser un recurso estratégico en el que las empresas necesitan herramientas para su obtención y análisis, de manera que se identifiquen los cambios del entorno y se tomen las acciones estratégicas para la organización.

Para esta investigación se utilizó la técnica de análisis envolvente de datos o también conocida como análisis de frontera, en la que se establecieron como variables de entrada el clima laboral, la gestión de la información y la gestión del conocimiento y como variables de salida que se optimizan, la productividad y la gestión de la innovación. Lo anterior permitió calcular el índice de eficiencia de un grupo de unidades empresariales pertenecientes al sector químico de la ciudad de Barranquilla, con los cuales se estructuró el índice de 
eficiencia $h_{o}$ de la unidad observada mediante la función objetivo de la ecuación (1) y sus condiciones restrictivas presentadas en la ecuación (2) (Visbal-Cadavid, et al, 2015).

$$
\operatorname{maxh}_{o}=\frac{\sum_{r=1}^{s} U_{r} * y_{r o}}{\sum_{i=1}^{m} V_{i} * x_{i o}}
$$

Sujeto a:

$$
\begin{aligned}
& \frac{\sum_{r=1}^{s} U_{r} * y_{r j}}{\sum_{i=1}^{m} V_{i} * x_{i j}} \leq \text { ó } \geq \text { ó }=1 \\
& \operatorname{con} j=1, \ldots, n
\end{aligned}
$$

donde s: número de variables de salida o resultado, $\mathrm{m}$ : número de variables de entrada o recurso, $U_{r}$ : peso relativo de la variable $r$-ésima de salida o resultado (positiva y desconocida), $y_{r o}$ : cantidad de la variable de salida r-ésima en la observación o, $V_{i}$ : peso relativo de la variable i-ésima de entrada o recurso (positiva y desconocida), $x_{i o}$ : cantidad de la variable de salida i-ésima en la observación o y $\mathrm{n}$ : cantidad de observaciones analizadas.

Charnes, et al (1978) diseñaron el Análisis Envolvente de Datos DEA para estudiar unidades tomadoras de decisión designadas DMU, para esto establecieron limites o fronteras de eficiencia de las unidades objeto de estudio con el fin de valorar las organizaciones objeto de estudio. De igual forma otras investigaciones han analizado la eficiencia asociada con indicadores y rubros financieros (Khalili et al., 2012). Otros estudios desarrollados por Ghiyasi (2017), Niknazar y Bourgault (2017) y Hanafizadeh, et al (2014) analizan otras variables en diferentes contextos y muestran la relevancia del análisis envolvente de datos para analizar la eficiencia y mirar la causalidad de como unas variables de entrada inciden en los resultados esperados de un grupo de unidades de toma de decisión. El análisis Discriminante Multivariado ADM, es una técnica de Análisis Multivariante que establece relaciones lineales entre las variables que mejor discriminan en las categorías de grupos previamente definidos (Elizondo y Altaman, 2003). Tiene como objetivo evaluar si existen diferencias significativas entre grupos de observaciones en relación a las variables analizadas de manera que se puedan proporcionar procedimientos de clasificación que permitan predecir la pertenencia de nuevas observaciones en uno de los grupos analizados (Mateos, et al. 2009).

Así mismo, según Bishop (2008), las Redes Neuronales Artificiales RNA es una técnica computacional basada en el proceso de aprendizaje con gran potencial de aplicación. Se basan en las características funcionales del sistema nervioso humano, utiliza un modelo de optimización no lineal (Miranda y Guzmán, 2017). Por su parte, McMillan (2013) conceptúa las RNA como redes compuestas de nodos de cómputo no lineal que incorporan una aproximación a las neuronas biológicas. Altamirano et al (2017) plantean que las RNA son estructuras capaces de imitar las funciones del cerebro humano para aprender relaciones complejas de datos, para identificar patrones y hacer predicciones acerca de nuevos datos, pueden usarse para determinar la pertenencia de un elemento desconocido a una categoría o para establecer un valor numérico especifico; su modelamiento implica la preparación de los datos, el entrenamiento y prueba de la red y la predicción de resultados. Otros autores como Hornik, et al (1989); Jiahe, et al (2003); Martín, et al (2007); Reddy (2005); Velásquez, et al (2009) lo asocian a un modelo matemático que imita de manera simplificada el procesamiento de información del cerebro. Matemáticamente, la sumatoria de los diferentes valores modificados por los pesos sinápticos, que determinan si la neurona se activa o no, se calcula mediante la ecuación 3.

$$
\text { Net }_{j}=\sum_{i=1}^{N} x_{i} w_{j i}+\theta_{j}
$$

Donde $N e t_{j}$ representa la entrada neta, $\mathbf{w}_{j i}$ el peso sináptico de la neurona $j$ sobre la entrada $x_{i}$.

La neurona se activa mediante una función de activación, la cual propaga la salida $y_{j}$ de la neurona hacia otra neurona o la salida de la red como se muestra en la ecuación 4 (Caicedo y López, 2009). En esta investigación se utiliza el perceptrón multicapa, el cual según Hernández et al. (2005) construye una red en forma de cascada con una o más capas ocultas.

$$
y_{j}=\operatorname{Fact}\left(\text { Net }_{j}\right)
$$


Comprende el conjunto de factores humanos y físicos en los que se desarrollan las actividades empresariales, los cuales son determinantes para la satisfacción laboral. Así, el ambiente laboral contribuye en el desempeño del trabajador especialmente en trabajos continuos y monótonos, teniendo efecto positivo sobre los factores de productividad (Rahmillah, 2016). Por el contrario, ambientes laborales con cargas de trabajo excesivas, condiciones deficientes en lo ergonómico, social y ambiental disminuyen la productividad del trabajador (Carvalho, et al 2017; Kim y Bong, 2017). Por otra parte, Santos y Martins (2018) plantean la necesidad de desarrollar la gestión de la información en el entorno organizacional a fin de mejorar los procesos diarios y la toma de decisiones estratégicas para el logro de ventajas competitivas y el éxito en los mercados. Devece, et al (2017) muestran la relación causal positiva de la gestión de la información y factores de rendimiento organizacional asociados a la posición competitiva, la productividad y la satisfacción del cliente. Así mismo, Dzenopoljac y Bontis (2018) plantean que procesos como la generación y desarrollo del conocimiento, codificación y almacenamiento, transferencia e intercambio asociados a la gestión del conocimiento tienen impacto positivo en la gestión comercial de la organización siendo relevante su impacto en el rendimiento de la innovación. Sharmila y Giuseppina (2018) plantean la necesidad de generar capacidad efectiva en la gestión del conocimiento para mejorar el desempeño general de las organizaciones empresariales. Según Phusavat, et al (2013), la competitividad de las organizaciones, requiere el uso productivo de los activos de la organización

\section{METODOLOGÍA}

Para el desarrollo de esta investigación se partió de información primaria tomada en el año 2016 de 52 empresas por medio de la aplicación de un instrumento validado que se aplicó a los directivos de rango medio y alto con conocimiento amplio de la organización (De la Hoz, et al. 2016). Respecto a las variables gestión de clima laboral, gestión de la información, la gestión del conocimiento, la gestión de indicadores de productividad e innovación en las empresas del sector químico, la validación del instrumento generó un Alfa de Cronbach de $97.7 \%$ de confiabilidad y un índice de validez de contenido de $91 \%$ de confiabilidad. En la figura 1 se presenta el diseño metodológico para valorar, clasificar y predecir la eficiencia técnica de las empresas analizadas.

Seguidamente se estableció la estructura DEA con las variables asociadas a los indicadores relacionados con la gestión del clima laboral, gestión de la información y gestión del conocimiento. Y como variables de salida se estableció la gestión de la productividad y la gestión de la innovación, lo anterior teniendo en cuenta que para esta investigación se analizó cómo dichos factores de entrada afectan e impactan en la productividad e innovación empresarial para competir en el medio, por lo que fue pertinente el modelo DEA CCRO orientado a la optimización de las variables de salida. Una vez identificada las empresas eficientes y no eficientes, con base al análisis Discriminante se analizó la pertenencia de estas o no a un grupo particular. Adicionalmente se procedió a establecer la estructura de la red neuronal que permitiera pronosticar y predecir la pertinencia de una empresa con las características establecidas en el modelo de Análisis Envolvente de Datos.

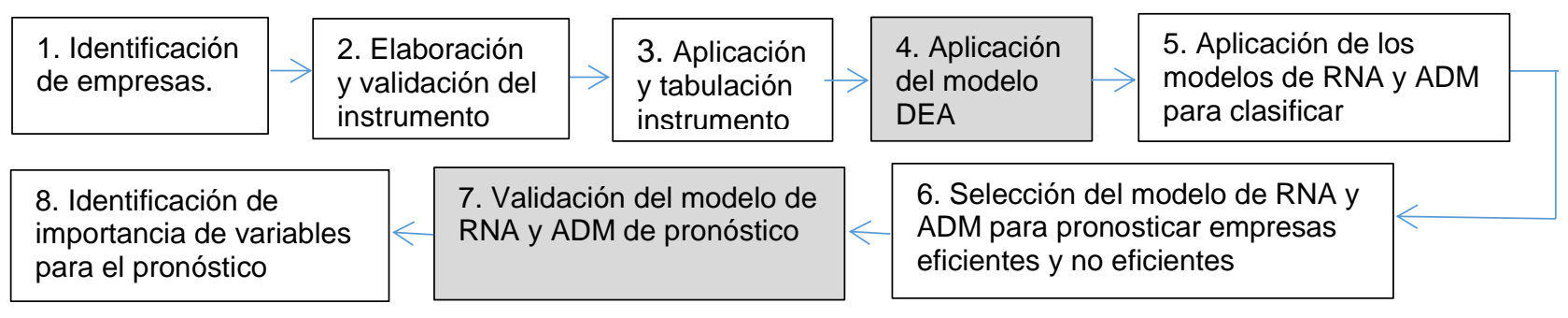

Fig. 1: Metodología para valorar, clasificar y predecir empresas eficientes y no eficientes

Para el análisis de las variables seleccionadas se trabajó el modelo DEA para lo cual se utilizó el software Dea Solver Pro versión 10 y para el análisis el estudio de redes Neuronales se utilizó IBM SPSS Statistics 25, cuya estructura de red implicó 5 variables estandarizadas en la entrada, 2 capas ocultas, con función de activación de tangente hiperbólica, en donde la variable de salida o dependiente es binaria con empresas eficientes y empresas no eficientes. Lo anterior permitió clasificar y agrupar las empresas del sector en función de la valoración de las eficiencias del sector químico de Barraquilla. Finalmente se validó el modelo propuesto para el pronóstico de empresas eficientes y no eficientes, con la aplicación de otras estructuras y modelos de redes neuronales que a través de evidencia empírica mostraron resultados iguales o muy similares.

\section{RESULTADOS}

Del análisis DEA se pudo establecer que 6 empresas de las 52 del sector químico que conformaban la población, mostraron ser eficientes en términos de la productividad e innovación. A continuación, en la Tabla 1 se presenta el Ranking de eficiencia de las pequeñas empresas del sector químico de Barranquilla. Lo cual 
permitió establecer la clasificación para analizar la pertenecía estadística a los dos grupos de empresas. Del estudio de Análisis envolvente de datos también se pudo evidenciar que el promedio de eficiencia solo alcanzo un $52,9 \%$, por tanto, esta investigación cobra importancia toda vez que con base en los indicadores calculados se puede establecer si potencialmente una empresa será o no eficiente en el sector en términos de productividad e innovación. Cuando se analizan como variables de entrada el clima laboral, la gestión de la información y gestión del conocimiento.

Tabla 1: Ranking de eficiencia de las pequeñas empresas del sector químico de Barranquilla

\begin{tabular}{|c|c|c|c|c|c|c|c|c|c|c|c|}
\hline Rank & DMU & Score & Rank & DMU & Score & Rank & DMU & Score & Rank & DMU & Score \\
\hline 1 & 46 & 1 & 14 & 41 & 0,6806 & 27 & 17 & 0,5694 & 40 & 21 & 0,4350 \\
\hline 1 & 36 & 1 & 15 & 47 & 0,6770 & 28 & 43 & 0,5600 & 41 & 29 & 0,4200 \\
\hline 1 & 24 & 1 & 16 & 23 & 0,6760 & 29 & 40 & 0,5342 & 42 & 30 & 0,4166 \\
\hline 1 & 3 & 1 & 17 & 32 & 0,6666 & 30 & 14 & 0,5312 & 43 & 9 & 0,4000 \\
\hline 1 & 18 & 1 & 18 & 2 & 0,6590 & 30 & 5 & 0,5312 & 43 & 28 & 0,4000 \\
\hline 1 & 12 & 1 & 19 & 1 & 0,6375 & 30 & 8 & 0,5312 & 45 & 31 & 0,3937 \\
\hline 7 & 6 & 0,8571 & 19 & 16 & 0,6375 & 33 & 51 & 0,5259 & 46 & 38 & 0,3693 \\
\hline 8 & 27 & 0,8305 & 21 & 52 & 0,6285 & 34 & 42 & 0,5250 & 47 & 45 & 0,3333 \\
\hline 9 & 13 & 0,8068 & 22 & 37 & 0,6250 & 35 & 20 & 0,5125 & 47 & 35 & 0,3333 \\
\hline 10 & 22 & 0,7968 & 23 & 15 & 0,6000 & 36 & 49 & 0,5000 & 47 & 11 & 0,3333 \\
\hline 11 & 48 & 0,7562 & 24 & 34 & 0,5833 & 37 & 50 & 0,4666 & 50 & 44 & 0,2500 \\
\hline 12 & 10 & 0,7500 & 24 & 4 & 0,5833 & 38 & 33 & 0,4583 & 51 & 39 & 0,1833 \\
\hline 13 & 26 & 0,7000 & 24 & 25 & 0,5833 & 38 & 7 & 0,4583 & 52 & 19 & 0,0700 \\
\hline
\end{tabular}

Para analizar la pertenencia de los grupos de empresas eficientes y no eficientes primeramente, se realizó un análisis de las variables mediante el coeficiente de correlación de Pearson, los resultados mostraron correlación bilateral significativa al nivel de 0,01 de la variable de salida Gestión de la innovación (GIN) con las variables de entrada Gestión de la información GI (0,452), Gestión del conocimiento GC $(0,450)$ y Gestión del clima laboral GCL $(0,460)$, mientras que no se evidenció correlación de las variables de entrada con la variable Gestión de productividad GP. Así mismo, se realizó un análisis discriminante que permitió establecer un promedio de clasificación de pertenecía correcta del 96,2 \% de las empresas del sector químico en la ciudad de Barranquilla. El modelo clasifica de forma correcta las empresas eficientes con una probabilidad de $83,3 \%$ y del 97,8 a las empresas no eficientes. Para un promedio del 96,2\% como se mencionó previamente. Lo cual es bueno considerando el tamaño de la muestra de las empresas objeto de la investigación, que fue pequeña. En la Tabla 2, se presentan los resultados de clasificación del análisis discriminante. Donde en el recuento, 1,00 representa a las empresas eficientes y 2,00 las no eficientes. En las ecuaciones 5 y 6 , se presentan las funciones discriminantes lineales de Fisher para los grupos de eficiencia, a partir del cual se puede pronosticar la pertenencia de empresas en los grupos analizados. Es importante señalar que otras investigaciones han mostrado la pertinencia de la técnica de Análisis discriminante para desarrollar procesos de clasificación y pertenecía de grupos empresariales como lo evidencias diferentes investigaciones (De La Hoz, et al, 2018; Fontalvo, 2014; y Fontalvo, et al, 2012).

Tabla 2: Resultados de clasificación ADM

\begin{tabular}{|c|c|c|c|c|c|}
\hline \multicolumn{6}{|c|}{ Resultados de clasificación } \\
\hline \multirow{2}{*}{\multicolumn{3}{|c|}{ Eficiencia }} & \multicolumn{2}{|c|}{ Pertenencia a grupos pronosticada } & \multirow[t]{2}{*}{ Total } \\
\hline & & & 1,00 & 2,00 & \\
\hline \multirow[t]{4}{*}{ Original } & \multirow[t]{2}{*}{ Recuento } & 1,00 & 5 & 1 & 6 \\
\hline & & 2,00 & 1 & 45 & 46 \\
\hline & \multirow[t]{2}{*}{$\%$} & 1,00 & 83,3 & 16,7 & 100,0 \\
\hline & & 2,00 & 2,2 & 97,8 & 100,0 \\
\hline
\end{tabular}

$Z_{\text {eficientes }}=3,185 \mathrm{E}-9 * I P 1-4,046 \mathrm{E}-10 *$ IP2 $-3,310 \mathrm{E}-9 *$ IP3 $+1,236 \mathrm{E}-9 *$ IP4 $-2,713 \mathrm{E}-9 *$ IP5 -

$1,233 \mathrm{E}-9 *$ IP6 + 7,131E $-9 *$ IP7 + 2,155E $-9 *$ IP8 $-3,026$

$Z_{\text {no eficientes }}=1,845 \mathrm{E}-9 * I P 1-1,254 \mathrm{E}-10 * \mathrm{IP} 2-9,402 \mathrm{E}-11 * \mathrm{IP} 3-4,602 \mathrm{E}-10 * \mathrm{IP} 4+1,563 \mathrm{E}-9 * \mathrm{IP} 5+$

$6,041 \mathrm{E}-10 *$ IP $-4,171 \mathrm{E}-11 *$ IP7 $-2,474 \mathrm{E}-10 *$ IP8 $-2,311$

\section{Modelo de red neuronal}

Para pronosticar la pertenencia de empresas eficientes y no eficientes en los resultados, se diseñó un modelo de Red Neuronal Artificial (RNA), mediante el software SPSS 16 en el que se utilizó una red de doble capa con función de entrada la Tangente Hiperbólica y como salida la función Sigmoide. En el diseño del modelo, de RNA, se utilizó la información de 52 empresas del sector químico del Departamento del Atlántico representada por las variables de entrada: gestión de la información, gestión del conocimiento, gestión del 
clima laboral, gestión de la productividad y gestión de la innovación. Como variable de salida o dependiente la clasificación en empresas eficientes y no eficientes. La muestra fue dividida tomando una partición del $60 \%$ de las empresas para el entrenamiento de la red, un $20 \%$ para la muestra de prueba y un $20 \%$ para la muestra de reserva. En la Figura 2 se muestra la estructura del modelo RNA con 5 parámetros en la entrada, 3 en la capa oculta y 2 en la capa de salida.

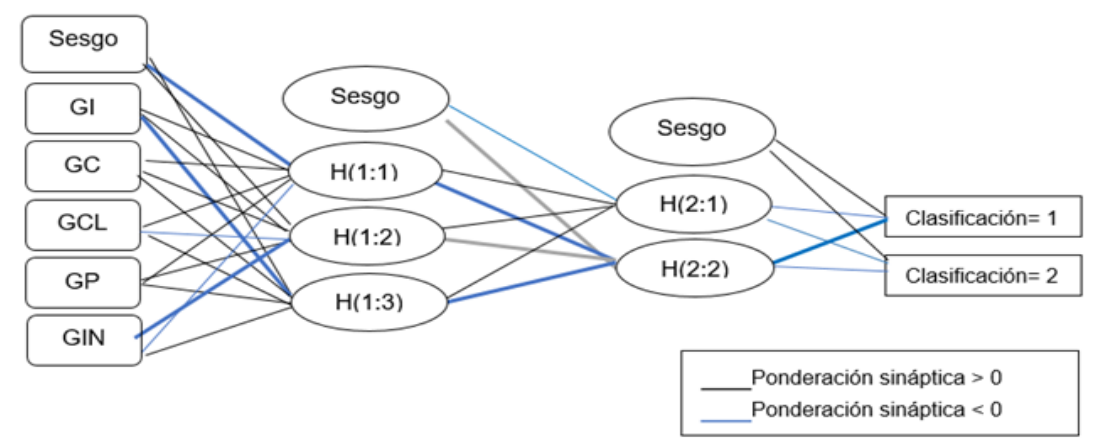

Fig. 2: Modelo de red neuronal para pronosticar la pertenencia de empresas eficientes y no eficientes.

En la Tabla 3 se observa que la estructura de red neuronal artificial propuesta, generó una precisión de predicción en la muestra de entrenamiento para las empresas eficientes del $100 \%$ y un $96,4 \%$ para las empresas no eficientes. En relación a la muestra de prueba la clasificación fue del $90 \%$ para las empresas de prueba. Y para la muestra de reserva la clasificación correcta de las empresas se da con una probabilidad del 100\%. En lo que sigue, se muestra una síntesis asociados con el análisis de pronóstico y predicción del método cuando se utiliza la técnica de cálculo multivalente de red Neuronal. La estructura pronostica considerando unas variables independientes asociadas al modelo DEA propuesto donde la variable dependiente o de clasificación se expresa por la eficiencia de las empresas (eficientes y no eficientes).

Tabla 3: Resultados de clasificación RNA

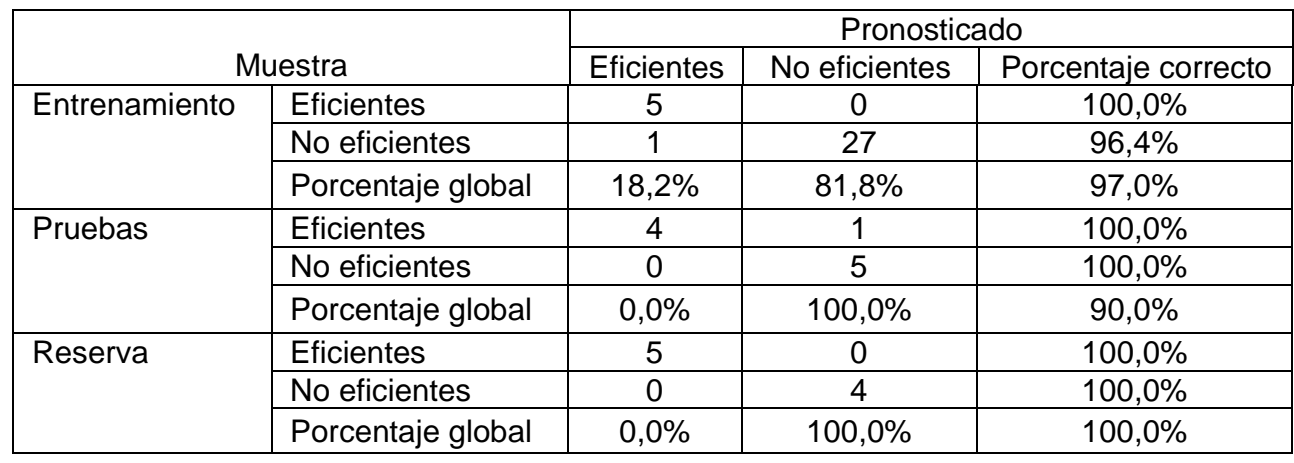

\section{Validación del modelo de redes neuronales de pronóstico}

Con el propósito de validar el modelo de red neuronal seleccionado para el proceso de pronóstico, se desarrolló un análisis de validación cruzada compuesto por 10 particiones con 9 observaciones cada una. Con lo anterior, se hicieron 5 corridas del modelo de RNA. En la Tabla 4, se muestran los resultados promedio de pronóstico en cada una de las muestras de entrenamiento, Prueba y Reserva, lográndose un 98,82\%, $95,78 \%$ y $94,28 \%$ de promedio porcentaje correcto de clasificación con lo cual se muestra la validez y relevancia del modelo de RNA. Tal y como lo muestran De La Hoz y López (2017).

Tabla 4: Validación cruzada pronóstico de clasificación correcta arquitectura RNA

\begin{tabular}{|c|c|c|c|c|c|c|c|}
\hline \multirow{2}{*}{ Muestra } & \multirow{2}{*}{ Observado } & \multicolumn{7}{|c|}{ Partición } \\
\cline { 2 - 7 } & & 1 & 2 & 3 & 4 & 5 & Promedio \% correcto \\
\hline \multirow{3}{*}{ Entrenamiento } & Eficientes & $100 \%$ & $100 \%$ & $100 \%$ & $100 \%$ & $100 \%$ & $100 \%$ \\
\cline { 2 - 7 } & No eficientes & $96,4 \%$ & $96,8 \%$ & $100 \%$ & $100 \%$ & $100 \%$ & $98,64 \%$ \\
\cline { 2 - 7 } & Porcentaje global & $97 \%$ & $97,1 \%$ & $100 \%$ & $100 \%$ & $100 \%$ & $98,82 \%$ \\
\hline \multirow{3}{*}{ Prueba } & Eficientes & $0 \%$ & $66,7 \%$ & $0 \%$ & $0 \%$ & $0 \%$ & $13,34 \%$ \\
\cline { 2 - 7 } & No eficientes & $100 \%$ & $100 \%$ & $100 \%$ & $100 \%$ & $100 \%$ & $100 \%$ \\
\cline { 2 - 7 } & Porcentaje global & $90 \%$ & $88,9 \%$ & $100 \%$ & $100 \%$ & $100 \%$ & $95,78 \%$ \\
\hline \multirow{3}{*}{ Reserva } & Eficientes & $0, \%$ & $0 \%$ & $0 \%$ & $100 \%$ & $0 \%$ & $20 \%$ \\
\cline { 2 - 7 } & No eficientes & $100 \%$ & $100 \%$ & $100 \%$ & $100 \%$ & $100 \%$ & $100 \%$ \\
\cline { 2 - 7 } & Porcentaje global & $100 \%$ & $100 \%$ & $85,7 \%$ & $100 \%$ & $85,7 \%$ & $94,28 \%$ \\
\hline
\end{tabular}


En la Tabla 5, se presentan los resultados de nivel de importancia de las variables analizadas, observándose como variables más importantes en el proceso de clasificación gestión del clima laboral GCL, gestión de productividad GP y gestión de la información GI.

Tabla 5: Nivel de importancia de las variables analizadas

\begin{tabular}{|l|c|c|}
\hline & Importancia & Importancia Normalizada \\
\hline Gestión de la información GI & 0,185 & $52,7 \%$ \\
\hline Gestión del conocimiento GC & 0,084 & $24,0 \%$ \\
\hline Gestión del clima laboral GCL & 0,352 & $100,0 \%$ \\
\hline Gestión de productividad GP & 0,272 & $77,4 \%$ \\
\hline Gestión de innovación GIN & 0,107 & $30,3 \%$ \\
\hline
\end{tabular}

\section{DISCUSIÓN FINAL}

En el análisis de las variables para evaluar la eficiencia de las empresas del sector químico de Barranquilla, se establecieron como variables de entrada, las variables de clima laboral, gestión de la información y la gestión del conocimiento y como variables de salida indicadores de productividad e innovación, las cuales mediante la técnica de análisis envolvente de datos permitió establecer el nivel de eficiencia de las empresas analizadas. Los resultaron mostraron una relación de eficiencia promedio del 52,9\%. Por su parte, la técnica de análisis discriminante multivariado permitió generar funciones discriminantes capaces de clasificar correctamente las empresas en eficientes y no eficientes, con un nivel de efectividad del $96,2 \%$. Así mismo cuando se estableció la red neuronal la precisión del modelo para predecir las empresas eficientes y no eficientes el modelo es relevante toda vez que generó una precisión del 98,82\%, 95,78\% y 94,28\% para las muestras de Entrenamiento, Prueba y Reserva. De igual forma esta investigación aporta a la comunidad científica un método que permite analizar cómo inciden las variables de clima laboral, gestión de la información y la gestión del conocimiento en los indicadores de productividad e innovación en las empresas del sector químico lo cual es replicable en cualquier otro contexto empresarial a nivel nacional o internacional

\section{CONCLUSIONES}

De acuerdo a los resultados obtenidos, se concluye:

1.- A partir del análisis envolvente de datos, se pudo establecer la relevancia del clima laboral, la gestión de la información y la gestión del conocimiento como variables de entrada y la gestión de productividad y la gestión de la innovación como variables de salida para valorar la eficiencia organizacional.

2.- Se pudo establecer relación de causalidad entre las variables Gestión de la información GI $(0,452)$, Gestión del conocimiento GC y Gestión del clima laboral GCL con la eficiencia en innovación de la organización. Así mismo en este estudio no se pudo establecer correlación de GI, GC y GCL con la eficiencia en la productividad

3.- Las variables analizadas clima laboral, gestión de la información y la gestión del conocimiento mostraron tener condiciones discriminantes para clasificar grupos de empresas eficientes y no eficientes lográndose un modelo con clasificación de pertenecía correcta del 96,2 \% de las empresas del sector químico en la ciudad de Barranquilla.

4.- Se demostró la validez y relevancia de la técnica de Redes Neuronales Artificiales para procesos predictivos clasificatorios, lográndose un modelo con una capacidad del 94,28\% para predecir de manera correcta la clasificación de empresas eficientes y no eficientes.

\section{REFERENCIAS}

Alegra, J., La gestión del conocimiento como motor de la innovación. Lecciones de la Industria de alta tecnología para la empresa. 1a Ed. 47-67, Editorial Athenea, Castellón de la Plana, España (2004)

Carvalho, D., L. Rocha y otros cuatro autores, Productivity versus workloads in the nursing working environment, doi: 10.1590/S1980-220X2017028903301, Rev. Esc. Enferm., USP, 51:e03301 (2017)

Cegarra, J. y A. Martínez, Gestión del conocimiento: Una ventaja competitiva. $1^{a}$ Ed., 19-20, Editorial ESIC, Madrid, España (2017)

Chiang, M., M. Martín y A. Núñez, Relaciones entre el Clima Organizacional y la Satisfacción Laboral, 1ª Ed., 14-15 Universidad Pontificia Comillas, Madrid, España (2010)

De La Hoz, E. y L. López, Aplicación de Técnicas de Análisis de Conglomerados y Redes Neuronales Artificiales en la Evaluación del Potencial Exportador de una Empresa, Información Tecnológica, 28 (4), 67-74 (2017) 
De La Hoz, E., A. González y A. Santana, Metodología de Medición del Potencial Exportador de las Organizaciones Empresariales, Información Tecnológica, 27 (6), 11-18 (2016)

De La Hoz, E., T. Fontalvo y J. Morelos, Diseño de Perfiles Financieros Empresariales del Sector Químico en Colombia mediante Cálculo Multivariado, doi: 10.4067/S0718-07642016000600003, Información Tecnológica, 29 (4), 197-204 (2018)

De La Peña, N., Gestión y control de los Sistemas de información, Ed. 5.1, 14-15, Editorial Elearning, S.L. España (2015)

De Pablos, C. y otros cinco autores, Dirección y gestión de los sistemas de información en la empresa: una visión integradora, 2 ${ }^{\mathrm{a}}$ Ed., 103-115, Editorial ESIC, Madrid, España (2006)

Devece, C., D. Palacios y D. Martinez-Simarro, Effect of information management capability on organizational performance, doi: 10.1007/s11628-016-0320-7, Service Business, 11(3), 563-580 (2017)

Dzenopoljac, V., R. Alasadi y N. Bontis, Impact of knowledge management processes on business performance: Evidence from Kuwait, doi: 10.1002/kpm.1562, knowledge and process management, 25(2), 77-87 (2018)

Elizondo, A. y E. Altman, Medición integral del riesgo de crédito, ISBN: 978-968-18-6358-6, Limusa, 200 p. (2003)

Fontalvo, T., Aplicación de análisis discriminante para evaluar la productividad como resultado de la certificación BASC en las empresas de la ciudad de Cartagena, Contaduría y administración 59 (1), 43-62 (2014)

Fontalvo, T., J. Vergara y E. De La Hoz, Evaluación del mejoramiento de los indicadores financieros en las empresas del sector almacenamiento y actividades conexas en Colombia por medio de análisis de discriminante, Prospectiva 10(1), 124-131 (2012)

Gan, F. y J. Trigine, Clima Laboral, 1aㅡ Ed., 275-276, Editorial Díaz de Santos, Colección: Monografías, serie: Administración/ Marketing, Madrid (2012)

Ghiyasi, M., Efficiency improvement and resource estimation: a tradeoff analysis, International, doi: 10.1504/IJPQM.2018.094758, Journal of Productivity and Quality Management, 25(2) (2018)

Hanafizadeh P., H. Reza y otros dos autores, Neural network DEA for measuring the efficiency of mutual funds, International Journal of Applied Decision Sciences, 7(3), 255-269 (2014)

Khalili-Damghani y M. Mohammad, Un enfoque difuso DEA de tres etapas para medir el rendimiento de un proceso en serie que incluye prácticas de JAT, índices de agilidad y objetivos en las cadenas de suministro. En t. J. of Services and Operations Management, 13(2), 147 - 188 (2012)

Kim, K. y S. Bong, Influences of Creative Personality and Working Environment on the Research Productivity of Business School Faculty, Creativity Research Journal, 29(1), 10-20 (2017)

López, J., +Productividad, $1^{\text {a }}$ Ed., 10-20, Editorial Palibrio, EE.UU (2013)

Manosalvas, C., L. Manosalvas y J. Nieves, El clima organizacional y la satisfacción laboral: un análisis cuantitativo riguroso de su relación, AD-minister, 26, 5-15 (2015)

Martínez, L., Gestión Del Cambio y la Innovación en la Empresa. Un modelo para la innovación, $1^{\text {a }}$ Ed., 1-15, Editorial Ideaspropias, Vigo, España (2006)

Mateos, R., J. iturrioz R. Gimeneo, La participación financiera y el papel de la mujer en la toma de decisiones de las sociedades cooperativas, Revista Europea de Dirección y Economía de la Empresa, 18(2), 65-82 (2009)

Niknazar, P. y M. Bourgault, Analysis of Axioms and Assumptions of Data Envelopment Analysis (DEA); Application for Efficiency Measurement in Project Management Contexts, doi: 10.1504/IJPQM.2018.090259, International Journal of Productivity and Quality Management (2018)

Phusavat, K., N. Comepa y otros dos autores, Productivity management: integrating the intellectual capital, doi: 10.1108/IMDS-09-2012-0330, Industrial Management y Data Systems, 113(6), 840-855 (2013)

Rahmillah, F., Optimization of Physical Working Environment Setting to Improve Productivity and Minimize Error by Taguchi and VIKOR Methods, doi:10.1088/1757-899X/105/1/012025, IOP Conf. Series: Materials Science and Engineering, 105 (2016)

Santos, B. y L. Martins, The information management and the information literacy: subsidies for business context, 7(1), (2017)

Segredo, A., Clima organizacional en la gestión del cambio para el desarrollo de la organización, Revista Cubana de Salud Pública, 39(2), 385- 393 (2013)

Serrate, A., Diagnosis of the working environment in an organization of scientific technological information, Revista Cubana de Información en Ciencias de la Salud, 25(1), 110-125 (2014)

Sharmila, G., E. Gianluca y P. Giuseppina, The effect of HRM practices on knowledge management capacity: a comparative study in Indian IT industry, doi: 10.1108/JKM-10-2017-0453, J. of Knowledge Management, 22(3), 649-677 (2018)

Visbal-Cadavid, D., A. Mendoza-Mendoza y K. Corredor-Carrascal, Evaluación del desempeño docente mediante análisis envolvente de datos: un estudio de caso, Revista Entramado, 11(2), 218-225 (2015) 